\title{
Promoted Osteoconduction of Polyurethane-Urea Based 3D Nanohybrid Scaffold through Nanohydroxyapatite Adorned Hierarchical Titanium Phosphate
}

Sanjoy Kumar Ghorai ${ }^{\dagger}$, Somnath Maji ${ }^{¥}$, Bhuvaneshwaran Subramanian ${ }^{\Psi}$, Tapas Kumar Maiti ${ }^{¥}$, and Santanu Chattopadhyay ${ }^{\dagger *}$

†Rubber Technology Centre, Indian Institute of Technology, Kharagpur-721302, India.

${ }^{\sharp}$ Department of Biotechnology, Indian Institute of Technology, Kharagpur-721302, India.

${ }^{\Psi}$ School of Medical science and Technology, Indian Institute of Technology, Kharagpur721302 , India.

*Corresponding author

Santanu Chattopadhyay; E-mail: santanuchat71@yahoo.com 
Table S1. Preparation of nHA adorned TP nanohybrid materials

\begin{tabular}{|c|c|c|}
\hline \multirow{2}{*}{$\begin{array}{c}\text { Designation of } \\
\text { Nanomaterials }\end{array}$} & \multicolumn{2}{|c|}{ Weight Percentage of nHA and TP } \\
\cline { 2 - 3 } & nHA $(\%)$ & TP $(\%)$ \\
\hline nHA & 100 & 0 \\
\hline nHA-20TP & 80 & 20 \\
\hline nHA-40TP & 60 & 40 \\
\hline nHA-60TP & 40 & 60 \\
\hline
\end{tabular}

Table S2. Synthesis of PUU based nanohybrid composites with pure nanohydroxyapatite and nanohybrid materials

\begin{tabular}{|c|c|c|}
\hline \multirow{2}{*}{$\begin{array}{c}\text { PUU Nanocomposites } \\
\text { (Scaffold) Designation }\end{array}$} & \multicolumn{2}{|c|}{ Filler Content (Weight Percentage) } \\
\cline { 2 - 3 } & nHA & nHA-TP \\
\hline PUU & $\ldots \ldots \ldots \ldots \ldots$ \\
\hline PUU/nHA & 1 & $\ldots \ldots \ldots$ \\
\hline PUU/nHA-20TP & $\ldots \ldots \ldots$ & 1 \\
\hline PUU/nHA-40TP & $\ldots \ldots \ldots$ & 1 \\
\hline PUU/nHA-60TP & $\ldots \ldots \ldots$ & 1 \\
\hline
\end{tabular}

Table S3. EDX study for elemental composition of synthesized nHA-TP nanohybrid materials

\begin{tabular}{|cccccccccc|}
\hline \multicolumn{2}{|c}{ Element Weight \% Atomic \% } & Net Int. & Error \% & Kratio & Z & R & A & F \\
OK & 33.16 & 53.14 & 154.82 & 13.58 & 0.11 & 1.12 & 0.95 & 0.29 & 1 \\
P K & 24.79 & 20.52 & 252.06 & 6.20 & 0.23 & 0.96 & 1 & 0.95 & 1.01 \\
CaK & 36.76 & 23.52 & 150.88 & 10.03 & 0.34 & 0.93 & 1.03 & 0.99 & 1.02 \\
TiK & 5.28 & 2.83 & 13.66 & 49.65 & 0.04 & 0.84 & 1.03 & 0.97 & 1.02 \\
\hline
\end{tabular}

Description S1. In vitro biomineralization study

In order to evaluate the osteoconductivity of the prepared nanohybrid scaffold, the in vitro biomineralization test was performed. The fabricated scaffold was dipped into the prepared simulated body fluid (SBF, prepare according to the Kokubo composition) and studied after 20 days of incubation. The morphology and the composition of the deposited mineralized layer were evaluated using FESEM and the attached Energy Dispersive X-Ray spectroscope (EDX, Inca X-sight, OXFORD Instrument). 

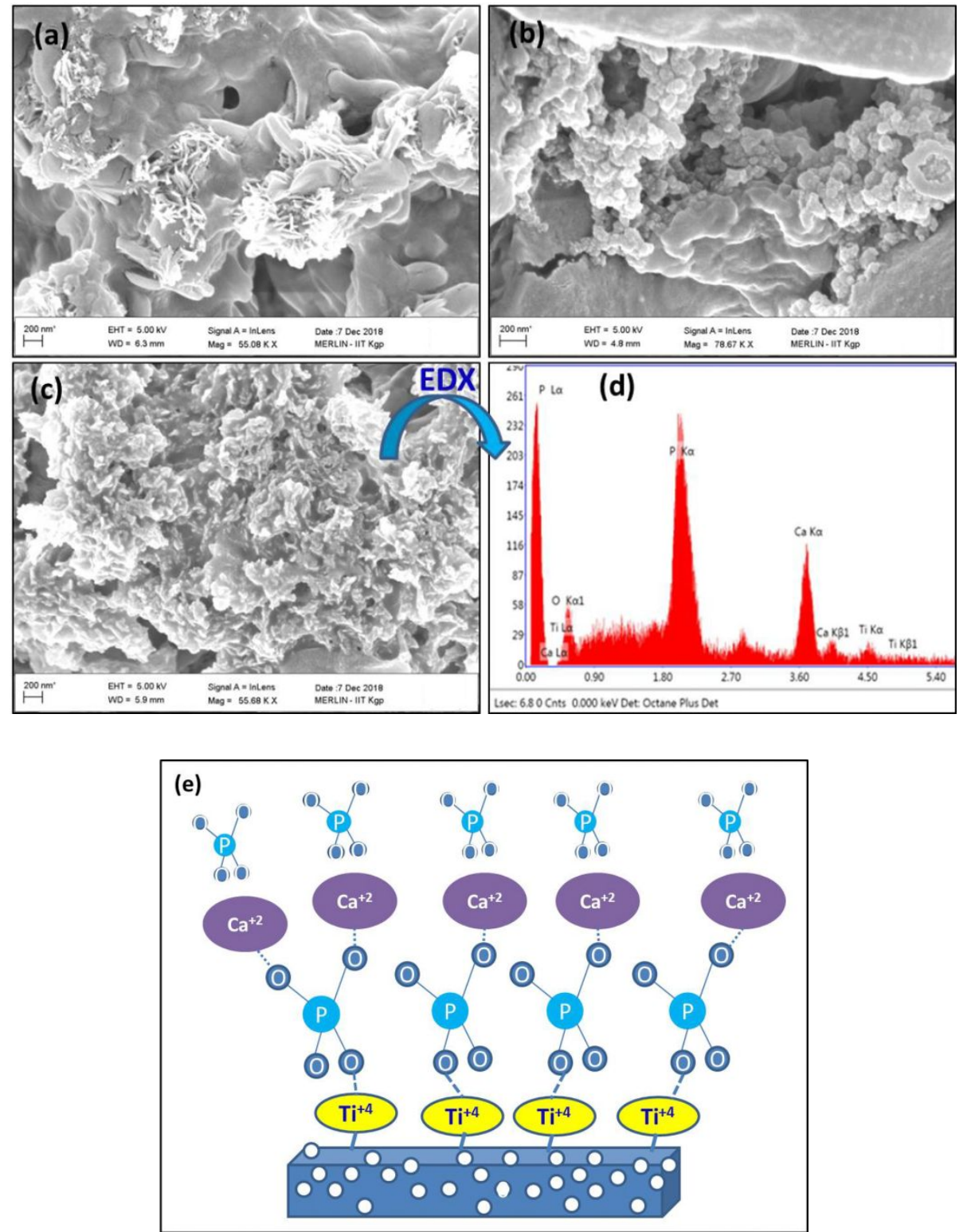

Figure S1. In vitro biomineralization study of PUU based nanohybrid scaffold after 20 days of incubation in SBF: (a) Pristine PUU, (b) PUU/nHA, (c) PUU/nHA-TP, (d) EDX study the deposited apatite layer, and (e) Represents the mechanism of formation of apatite layer on the scaffold by ionic interaction between the scaffold and the solvated ions. 
Description S2. Study of hydrophilic nature of PUU based scaffold through contact angle measurement

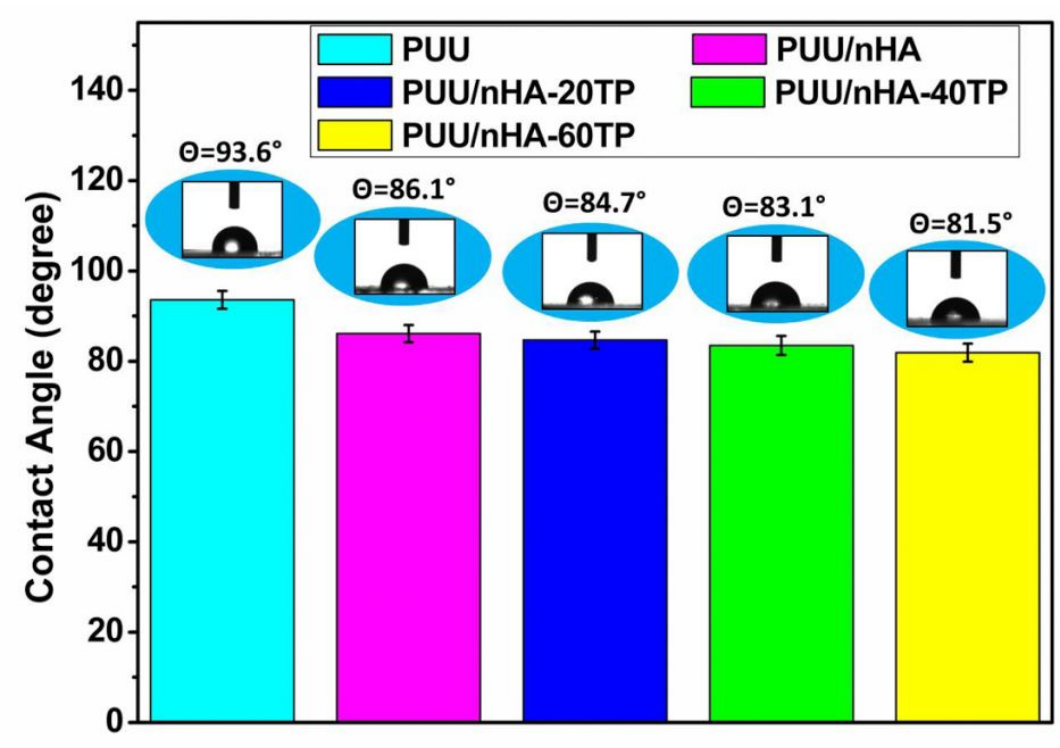

Figure S2. Contact angle measurement of pristine PUU and nanohybrid scaffolds.

Description S3. Fluorescent images of proliferated cells on the scaffolds

The morphology of the proliferated cells is visualized in a fluorescence microscope using the Calcein-AM staining agent. The images are captured after 3 days and 7 days of proliferation, as shown in Figure S3. Fluorescent images indicated that the seeded cells are well proliferated all over the scaffolds after 3 days, and it increases progressively after 7 days of culture. The nanohybrid scaffold PUU/nHA-40TP shows the maximum number of proliferated cells with cell-cell contact without much void space compare to the pristine and other scaffolds after 7 days of culture. The optimum level of adorned TP with the nHA, containing calcium and phosphate moieties accelerates the biomineralization and mimics the ECM structure, which favored the cell adhesion and proliferation. 

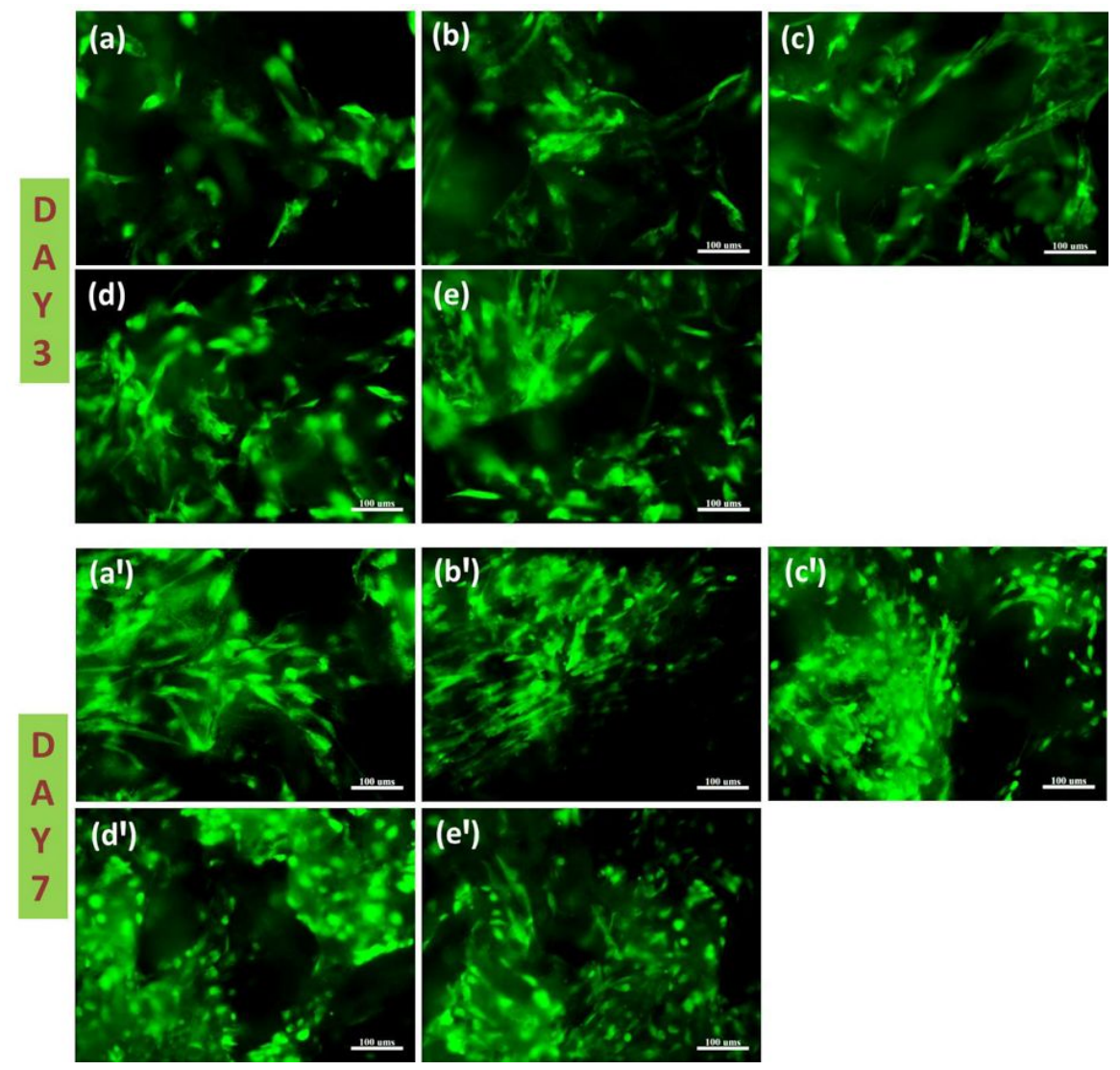

Figure S3. Fluorescent images of proliferated MG-63 cells after 3 days and 7 days of culture on different scaffolds. (a, a') Pristine PUU, (b, b') PUU/nHA, (c, c') PUU/nHA-20TP, (d, d') PUU/nHA-40TP, and (e, e') PUU/nHA-60TP.

\section{Description S4. Antibacterial study}

The antibacterial effect of the scaffolds, against gram-negative pathogen such as E. Coli (MTCC2939) was evaluated by agar disc diffusion method. The culture was stocked at $-80{ }^{\circ} \mathrm{C}$ using glycerol. The isolated strain was sub-cultured at $30{ }^{\circ} \mathrm{C}$ for $24 \mathrm{~h}$ in a nutrient broth. The strain was swabbed uniformly in the nutrient agar plate using a cotton swab. Subsequently, the scaffold was cut into a disc of $0.6 \mathrm{~mm}$ diameter and washed properly in PBS. The prepared discs were sterilized by $70 \%$ ethanol and UV light for 20 minutes and impregnated in gentamicin solution $(5 \mathrm{mg} / \mathrm{mL})$ of $100 \mu \mathrm{L}$. The discs were taken out from the solution and the excess solution was wiped filter paper and air dried for $4 \mathrm{~h}$. After that, the discs were placed on the prepared agar plate in an incubator at $37^{\circ} \mathrm{C}$ for $24 \mathrm{~h}$ and the inhibition zone was calculated using Image J software. 


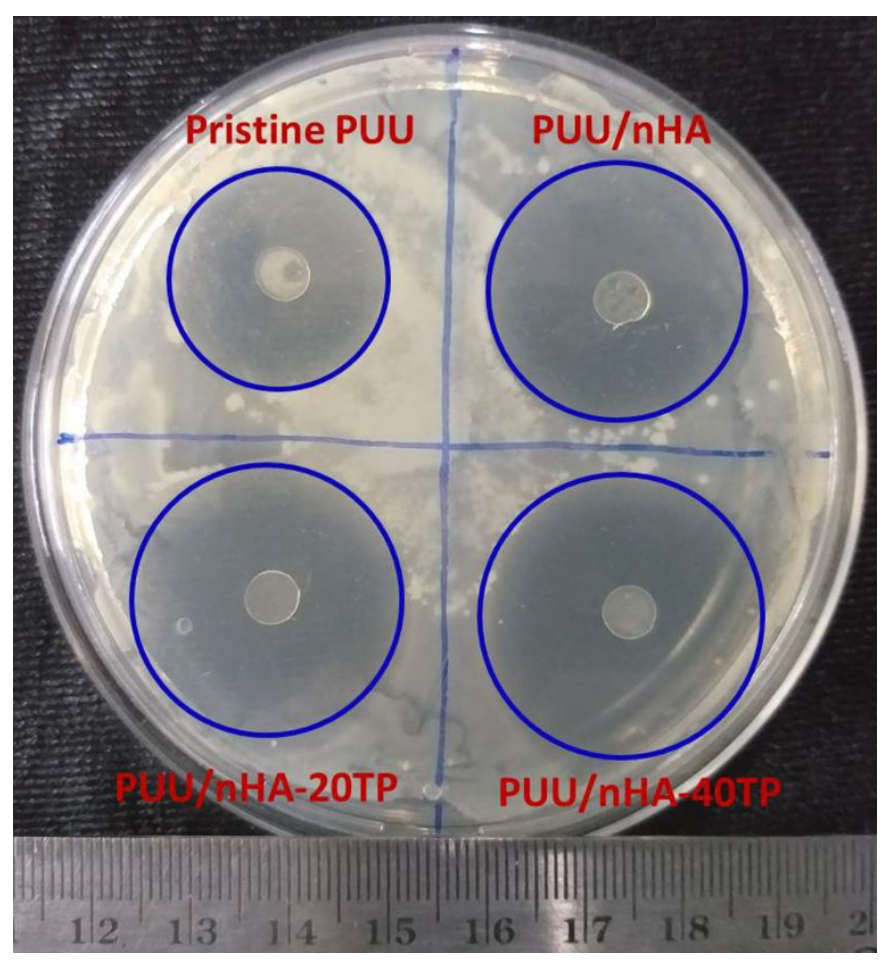

Figure S4. Antibacterial study of PUU based nanohybrid scaffolds against gram-negative pathogens E. Coli using agar disc diffusion method.

The bacterial infections are the most common problem in bone grafting surgery associated with the different pathogen such as E. Coli, streptococcus (not done here). Thus, the antibacterial study of bone grafting material is quite essential. The antibacterial effect of the synthesized scaffold increases by the addition of the nanomaterial within the polymer matrix. The PUU/nHA shows the zone of inhibition of diameter about $2.57 \mathrm{~cm}$ compares to the pristine PUU with the zone of inhibition of $2.20 \mathrm{~cm}$. The nHA adorned TP showed a higher value of zone of inhibition with the value of $2.71 \mathrm{~cm}$ and $2.84 \mathrm{~cm}$ for PUU/nHA-20TP and PUU/nHA-40TP, respectively. With the addition of nHA, the antibacterial property of the scaffold increases which is more pronounced in the presence of nHA adorned TP. The nHA and nHA-TP acted synergistically against the pathogens. ${ }^{1}$ The sharp edge of the nHA nanorod and the hierarchical structure of TP may act as a sharp cutter which cut the bacterial membrane causing the discharge the intracellular components and leads to cell death. ${ }^{2}$ Another reason for cells death can be interpreted by considering the formation of reactive oxygen species (ROS) including $\mathrm{HO}, \mathrm{HO}_{2}, \mathrm{O}_{2}{ }^{-}$during the sterilization in UV light. These species have the power to oxidized the cellular components and causing the lysis of bacteria, adhere to the nanomaterial. ${ }^{3}$ Although the antibacterial activity in the absence of UV irradiation is not properly clear though several mechanisms have been proposed. ${ }^{4}$ One plausible proposed mechanism is that the nanoparticle is adsorbed on the bacterial cell 
surface resulting nanoparticle-cell physico-mechanical interactions that produce oxidative stress on the cell via red-ox reaction. The TP nanoparticle oxidized the lipid and protein of the bacterial cell by generating the conducive bridge through the insulating lipid bilayer and oxidized the cellular components by accelerating the transfer of an electron from the intracellular component of the bacteria to the outer attached nanoparticle. Thus, the $\mathrm{Ti}^{+4}$ are reduced to $\mathrm{Ti}^{+3}$ or $\mathrm{Ti}^{+2}$ and causing oxidative degradation of cell membrane which leads to excretion of intracellular components, casing bacterial cell death. ${ }^{5}$

\section{Description S5. Compressive strength of the PUU and its nanocomposites}

The compressive strength of the synthesized PUU and PUU based nanocomposites are evaluated by preparing the cylindrical shape specimens of diameter $18 \mathrm{~mm}$ and length of 24 mm using the compression mold. The compressive strength of the material is measured at a cross-head speed of $20 \mathrm{~mm} / \mathrm{min}$. The compressive strength of the PUU/nHA-20TP shows the maximum compressive strength of $12 \mathrm{MPa}$ at a strain of $25 \%$.

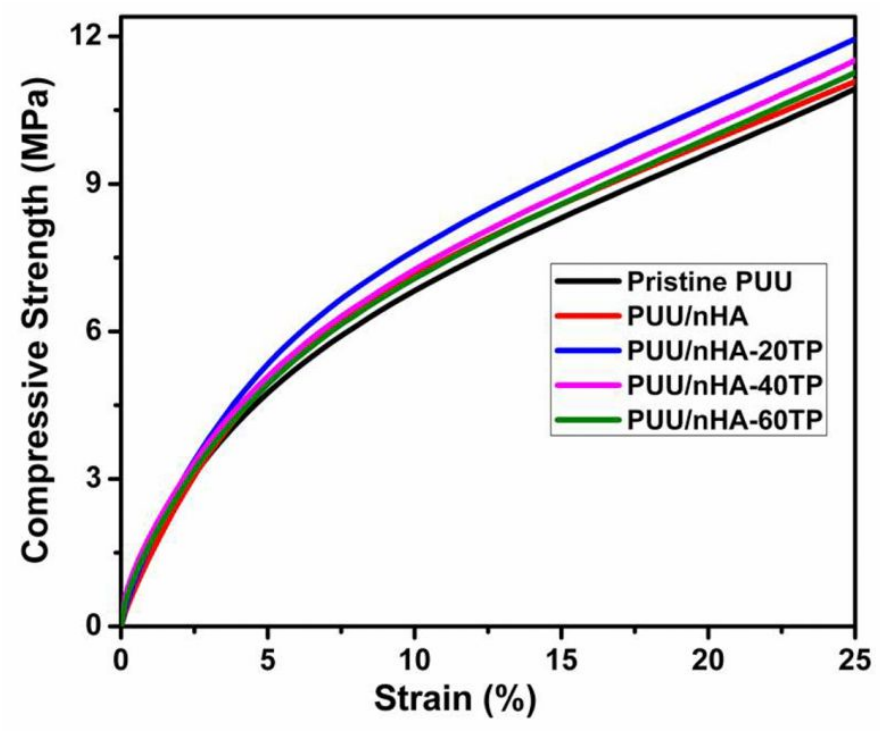

Figure S5. Compressive strength of the prepared nanohybrid composites at room temperature 


\section{Description S6. 3D Optical Surface Profilometry (3D OSP) study}

The surface roughness of the fabricated porous scaffold is determined by 3D Optical Surface Prifilometer. The rough surface of the fabricated scaffold helps to adhere the seeded cells on the scaffold. The root mean micro-roughness $(\mathrm{Rq})$ value of the scaffold is approximately 6.8 $\mu \mathrm{m}$.
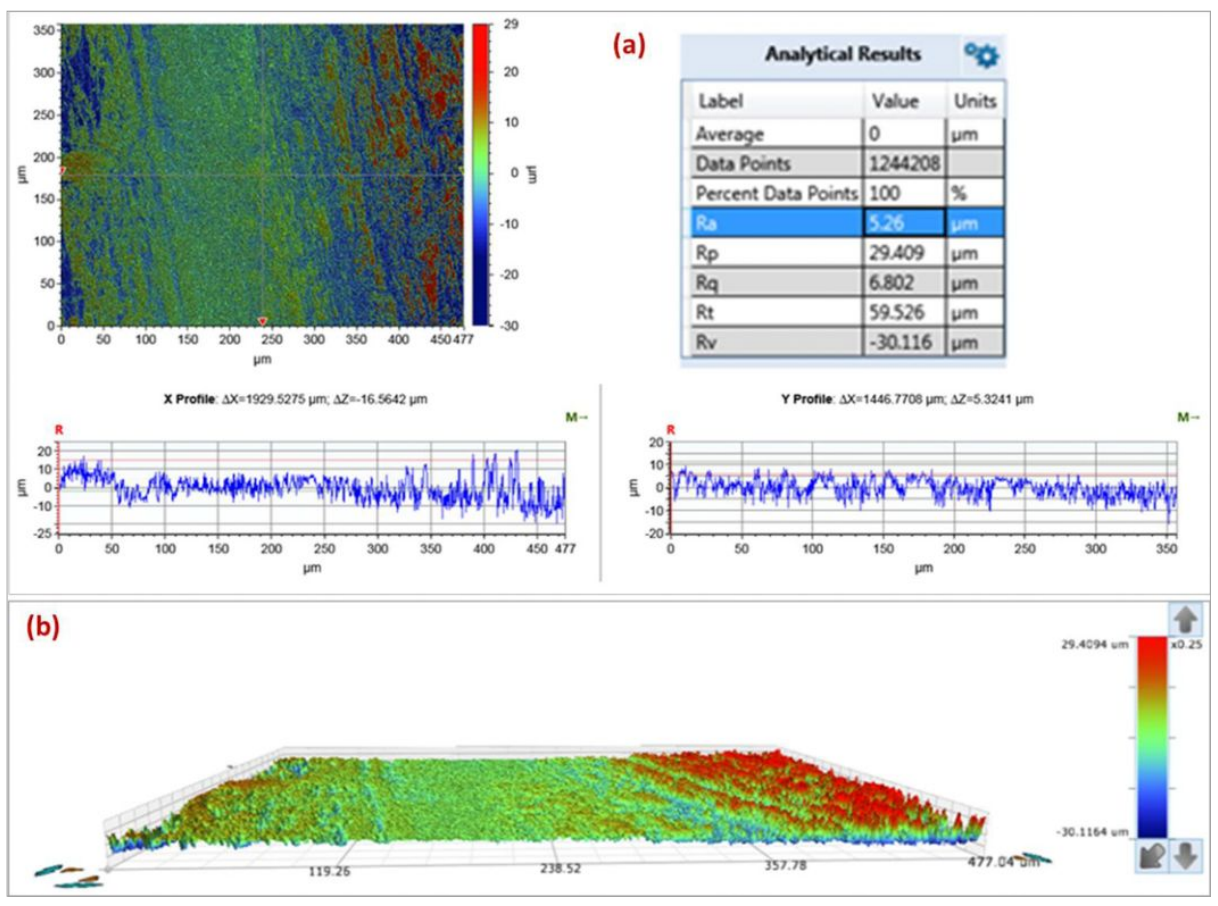

Figure S6. 3D Optical Surface Profilometry (3D OSP) study: (a) 2D OSP image, and (b) 3D OSP image of scaffold.

Description S7. FESEM morphology of seeded MG-63 cell on the scaffolds

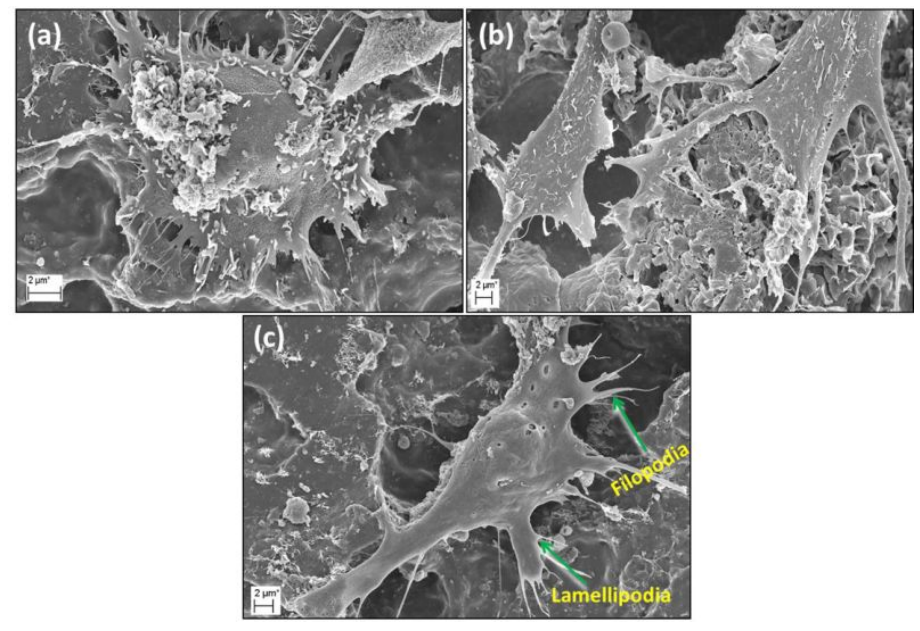

Figure S7. FESEM morphology of the seeded cell on the scaffold: (a) Pristine PUU, (b) PUU/nHA, and (c) PUU/nHA-40TP 
Description S8. Micro-CT images of the retrieved bone after 60 days of implantation

The extent of bone healing of the operated bone is calculated by using micro-CT images of the retrieved bone samples. ImageJ software is used to calculate the percentage of healing. The percentage healing of the bone is calculated as-

$$
\% \text { Healing }=\frac{\text { Area of the healed bone }}{\text { Initial area of the operated bone }} \times 100
$$

The percentage healing of the scaffold implanted operated bone is calculated. The control bone (without scaffold) and the PUU/nHA scaffold implanted bone sample shows $64 \%$ and $78 \%$ healing. Whereas the nanohybrid scaffold (PUU/nHA-40TP) implanted bone shows the maximum healing of $90 \%$.

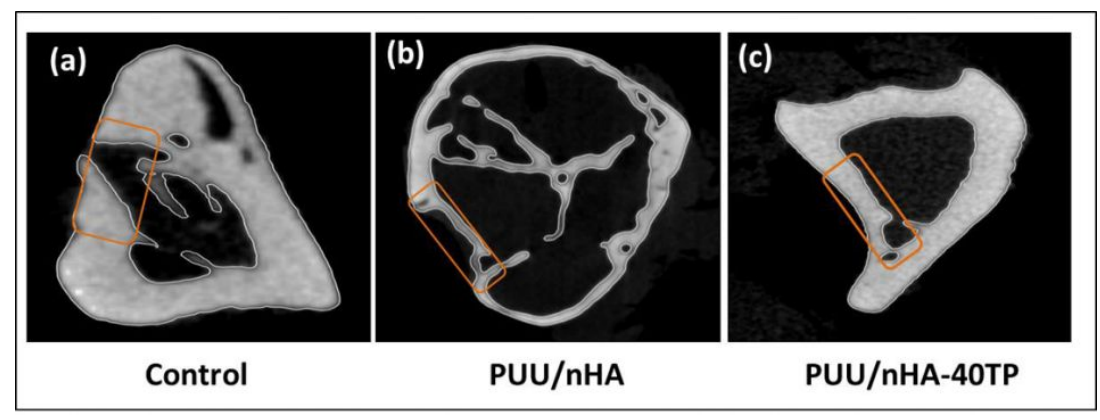

Figure S8. Micro-CT images of the retrieved bone after 60 days of implantation: (a) Control, (b) PUU/nHA, and (C) PUU/nHA-40TP.

\section{References}

(1) Wu, V. M.; Tang, S.; Uskoković, V. Calcium Phosphate Nanoparticles as Intrinsic Inorganic Antimicrobials: The Antibacterial Effect. ACS applied materials \& interfaces 2018, 10 (40), 34013-34028.

(2) Kapat, K.; Maity, P. P.; Rameshbabu, A. P.; Srivas, P. K.; Majumdar, P.; Dhara, S. Simultaneous hydrothermal bioactivation with nano-topographic modulation of porous titanium alloys towards enhanced osteogenic and antimicrobial responses. Journal of Materials Chemistry B 2018, 6 (18), 2877-2893.

(3) Díez-Pascual, A. M.; Díez-Vicente, A. L. Nano-TiO2 reinforced PEEK/PEI blends as biomaterials for load-bearing implant applications. ACS applied materials \& interfaces 2015, 7 (9), 5561-5573.

(4) Dalai, S.; Pakrashi, S.; Chakravarty, S.; Hussain, S.; Chandrasekaran, N.; Mukherjee, A. Studies on interfacial interactions of TiO 2 nanoparticles with bacterial cells under light and dark conditions. Bulletin of materials science 2014, 37 (3), 371-381. 
(5) Díez-Pascual, A. M.; Díez-Vicente, A. L. Effect of TiO 2 nanoparticles on the performance of polyphenylsulfone biomaterial for orthopaedic implants. Journal of Materials Chemistry B 2014, 2 (43), 7502-7514. 